\title{
Antibiotic use prior to a lung cancer diagnosis: a population-based study
}

\author{
Lukas Löfling $^{1,2}\left(\mathbb{0} \cdot\right.$ Shahram Bahmanyar ${ }^{1} \cdot$ Helle Kieler $^{1,3} \cdot$ Mats Lambe $^{4,5} \cdot$ Gunnar Wagenius $^{6}$
}

Received: 20 October 2020 / Accepted: 2 March 2021 / Published online: 22 March 2021

(c) The Author(s) 2021

\begin{abstract}
Aim To examine patterns of recent pre-diagnostic fillings of antibiotics as an indicator of early symptoms of lung cancer. Methods Individuals diagnosed with lung cancer (cases) in 2009-2016 were identified in the Swedish National Lung Cancer Register, a population-based register, and randomly matched with up to five individuals free of lung cancer (controls) from the general population. Conditional logistic models were used to estimate odds ratios for the association between lung cancer and a recent history of filled antibiotic prescriptions.

Results The study included 27,017 cases and 129,355 controls. The likelihood of recent exposure was approximately two times higher in cases compared to controls. The magnitude of the effect size became more pronounced with proximity to the diagnosis of lung cancer and an increasing number of filled prescriptions. While the magnitude of the effect size did not differ by sex or educational level, it became attenuated with increasing age. There was no evidence supporting a trend in the magnitude of the effect size for the association between lung cancer and a history of repeated fillings by cancer stage.

Conclusion Lung cancer was associated with an increased likelihood of a recent history of filled antibiotic prescriptions. However, there was no evidence of an association between repeated fillings and a diagnostic delay, as reflected by stage. Our findings underscore the importance of clinical reassessment to rule out lung cancer following pneumonia treatment, especially for patients with multiple treatment cycles.
\end{abstract}

Keywords Lung cancer $\cdot$ Antibiotics $\cdot$ Early symptoms $\cdot$ Sweden $\cdot$ Pneumonia

\section{Introduction}

At present, screening for lung cancer is only recommended in three high-income countries (United States of America, Canada, and Japan), focusing mainly on high-risk groups

Lukas Löfling

lukas.loflling@ki.se

1 Centre for Pharmacoepidemiology, Division of Clinical Epidemiology, Department of Medicine - Solna, Karolinska Institutet, Solna, Sweden

2 Etiological Research Unit, Department of Research, Cancer Registry of Norway, Oslo, Norway

3 Clinical Pharmacology, Department of Laboratory Medicine, Karolinska Institutet, Huddinge, Sweden

4 Regional Cancer Centre Central Sweden, Uppsala, Sweden

5 Department of Medical Epidemiology and Biostatistics, Karolinska Institutet, Solna, Sweden

6 Cancer Theme, Karolinska University Hospital, Solna, Sweden such as long-term smokers [1]. Today, approximately 50\% of patients with lung cancer in Western countries are diagnosed with late-stage cancer [2]. Because pneumonia can be an early symptom and is the most common differential diagnosis [3, 4], it is of interest to examine patterns of antibiotic use prior to the diagnosis of lung cancer.

The few studies to date that have examined histories of antibiotic use in patients with lung cancer have mainly focused on possible causal associations, i.e., antibiotics as a risk factor or risk modifier [5, 6]. In these studies, information on antibiotic use in the period directly before the date of lung cancer diagnosis has generally been excluded to avoid the influence of reversed causality.

Using routinely collected data, we aimed to compare patterns of recent fillings of prescriptions of antibiotics (i.e., within three years prior to the diagnosis of lung cancer) recommended for the treatment of pneumonia between patients with lung cancer and control individuals free of lung cancer. We also explored possible trends in the magnitude of the effect size for the association between 
a history of repeated pre-diagnostic fillings of antibiotic prescriptions and lung cancer by stage as a possible indicator of diagnostic delays.

\section{Materials and methods}

\section{Study design and study population}

We performed a population-based study with routinely collected data to examine possible associations between a diagnosis of lung cancer and a recent history of filled prescriptions of antibiotics recommended for the treatment of pneumonia (Supplementary Table S1). We identified individuals diagnosed with lung cancer (International Classification of Diseases for Oncology third edition [ICD-O-3] code: C34 or ICD-7 code: 1,621) between 2009 and 2016 (cases), as recorded in the Swedish National Lung Cancer Register (NLCR). The NLCR includes $97 \%$ of all individuals registered with a diagnosis of lung cancer in the Swedish Cancer Register (SCR) to which reporting is mandated [7, 8]. Using a risk set sampling approach, each case was individually matched with up to five randomly selected individuals free of lung cancer (controls), at the date of the diagnosis for the case, from the Swedish Population Register [9]. The matching factors were the year of birth, sex, and place of residence at the date of diagnosis for the case. The index date was defined as the date of the first lung cancer diagnosis recorded in the NLCR or the SCR, and the corresponding date for the matched controls.

We excluded cases with unclassified, unknown, or missing lung cancer histopathology, cases with more than a sixmonth difference between the recorded date of diagnosis in the NLCR and the SCR, and controls with a record of a lung cancer diagnosis in the SCR before the index date.

\section{Data sources}

Data for the present study were retrieved from the Lung Cancer Database Sweden (LCBaSe) [2, 10], a register-based research database generated by record linkages between the NLCR, the SCR, the National Patient Register (NPR), the Prescribed Drug Register (PDR), the Cause of Death Register (CDR), the Multi-generation Register (MGR), the Swedish Population Register, and the LISA-database (a database containing sociodemographic data). The record linkages were made possible by the use of the personal identity number, a unique personal identifier given to all residents of Sweden [9]. In this study, we used data in the LCBaSe from the NLCR, the SCR, the NPR, the PDR, the Swedish Population Register, and the LISA-database [8, 9, 11-14].

\section{Definition of exposure}

We used four different measures to assess the history of filled prescriptions of antibiotics recommended for treatment of pneumonia (Supplementary Table S1) as recorded in the PDR within three years prior to the index date:

(1) at least one filled prescription,

(2) repeated fillings ( $\geq 2$ fillings),

(3) the number of filled prescriptions (categorized as 0,1 , 2,3 , and $\geq 4$ ), and

(4) the number of different types of antibiotics (unique anatomical therapeutic chemical codes at the 5th level, categorized as $0,1,2$, and $\geq 3$ ).

\section{Statistical analysis}

Descriptive analyses were used to describe the clinical characteristics and demographics of cases and controls. Continuous variables were summarized using the median and the first and third quartiles (q1 and q3). Categorical variables were summarized with counts and per cent.

We used conditional logistic regression models, with the matched individuals as the clusters, to estimate odds ratios (OR) and 95\% confidence intervals (CI) for possible associations between a diagnosis of lung cancer (non-small cell lung cancer [NSCLC] overall, squamous cell carcinoma [SCC], adenocarcinoma, and small cell lung cancer [SCLC]) and a history of recent exposure. The effect of being a patient with lung cancer was estimated for the different histopathological subtypes overall and, within each histology, separately by sex, age, educational level, summarized cancer stage according to the tumor-node-metastasis [TNM] system from the American Joint Committee on Cancer [15], and separately for the descriptors that make up the stage. In the adjusted model, the highest attained educational level the year before the index date (categorized by years of formal education: $\leq 9,10-12$, and $\geq 13$; baseline level: " $\leq 9$ ") was used as an indicator of socioeconomic status. Other covariates included in the adjusted models were a record in the NPR of a chronic obstructive pulmonary disease (COPD) diagnosis (ICD-10: J44, baseline level: "no") in the five years prior to the index date, at least one filled prescription of pre-defined antibiotics at any time prior to the start of the observation period (a minimum of six months) (baseline level: "no"), a history of any cancer (any record of a diagnosis in the SCR prior to the index date) (baseline level: "no"), and the other TNM-descriptors in the descriptor specific subanalyses (e.g., if estimating the effect of exposure in subgroups of the T-descriptor then the estimate was adjusted for the $\mathrm{N}$ - and M-descriptors; baseline levels: "T1" and "N1"). 
We performed several sets of sensitivity analyses. First for different time intervals of the observation period, and second for those with an index date of the 1st July 2009 or after and without records of filled prescriptions of a predefined antibiotic within 12 months prior to the start of the observation period (washout period of 12 months) (Supplementary Figure S1). Third, where filled prescriptions in the three months before the index date were not considered, and fourth, following the exclusion of individuals with a history of COPD.

All data management and statistical analyses were performed using R statistical packages version 3.5.3 (R Development Core Team) and Stata version 15 (StataCorp LP), or later versions.

\section{Results}

We identified a total of 29,890 patients with a diagnosis of lung cancer between 2009 and 2016 (Supplementary Figure S2). Of these, 2,873 cases (and their matched controls) were excluded because of unclassified, unknown or missing histopathology $(n=2275)$ or more than a six-month difference between the recorded date of diagnosis in the NLCR and the SCR $(n=598)$. An additional 90 controls were excluded because of a record of diagnosis of lung cancer in the SCR before their index date. Hence, the final study population encompassed a total of 27,017 patients with lung cancer and 12,9355 controls.

\section{Characteristics of the study population}

A high educational level (i.e., post-upper secondary degree) was less common among cases (16\%) compared to controls (25\%) (Table 1). Compared to cases, a lower proportion of controls had a history of COPD (13\% and 3\%, respectively).

\section{Patterns and odds of fillings}

Half of the cases and one-third of the controls had a recent history of at least one filled prescription of antibiotics (Table 1). The proportion of cases and controls exposed to antibiotics did not vary substantially by age. For cases, the proportion varied between $47 \%$ ( $\geq 80$ years) and $52 \%$ (60-69 years), and for controls, between $30 \%$ ( $<50$ years) and $34 \%$ (60-69 years). Among patients with lung cancer, the percentage with at least one filled prescription started to increase around three to four months prior to the diagnosis, while it remained relatively stable for individuals free of lung cancer, this was independent of a history of COPD (Fig. 1). In patients with lung cancer, ever-smokers had more often filled at least one prescription compared to never-smokers, an observation that was present throughout the observation period (Supplementary Figure S3). The proportion of individuals with repeated fillings was $25 \%$ among patients with lung cancer and $13 \%$ among individuals free of lung cancer (Table 1), and the maximum treatment cycles were 63 and 78, respectively. In a comparison between individuals with and without a history of COPD, both cases and controls with COPD had more often filled repeated prescriptions of antibiotics (Supplementary Figure S4).

Compared to controls, the likelihood of a recent history of at least one filled prescription was elevated in patients with lung cancer: NSCLC overall (OR: 1.83, 95\% CI 1.77-1.88), SCC (OR: 2.18, 95\% CI 2.05-2.32), adenocarcinoma (OR: 1.70, 95\% CI 1.64-1.77), and SCLC (OR: 1.92, 95\% CI 1.78-2.06) (Table 2). The magnitudes of these effect sizes became more pronounced with an increasing number of filled prescriptions (Fig. 2) and with proximity to the diagnosis of lung cancer (Fig. 3, Supplementary Table S2). While the magnitudes of the effect sizes did not differ by sex or educational level, they became attenuated with increasing age (Table 2). Compared to controls, patients with lung cancer had between two and three times higher odds for a recent history of four or more filled prescriptions in three years prior to the diagnosis. A similar pattern was observed for the association with the number of different types of antibiotics from filled prescriptions (Supplementary Table S3).

For repeated fillings, we did not find a trend in the magnitude of the effect size by cancer stage (Table 3). However, there was an indication of a slightly pronounced magnitude of the effect size for stage III compared to stage IV disease. In separate assessments, there was no trend in the effect size by the T- and N-descriptors.

When assessing the exposure in the six months prior to the diagnosis only, the magnitudes of the ORs for repeated fillings became substantially pronounced compared to those unexposed and those who filled one prescription only, a pattern also present when assessing the association for different types of antibiotics from filled prescriptions (Supplementary Table S4, Supplementary Table S5). When we applied a 12-month washout period, the results remained essentially unchanged with an estimated two-fold increase in odds for a history of at least one filled prescription: NSCLC overall (OR: 1.84, 95\% CI 1.77-1.90), SCC (OR: 2.11, 95\% CI 1.96-2.27), adenocarcinoma (OR: 1.73 , 95\% CI 1.66-1.81), and SCLC (OR: 1.95, 95\% CI 1.78-2.13) (Supplementary Table S6 When excluding the three months closest before the index date, the magnitudes of the ORs decreased (Supplementary Table S7). However, an indication of more pronounced ORs with an increasing number of fillings remained. Following the exclusion of individuals with a history of COPD, the estimated ORs remained virtually unchanged (Supplementary Table S8). 
Table 1 Baseline characteristics of patients with a diagnosis of lung cancer (cases) and individuals free of lung cancer (controls), Lung Cancer DataBase Sweden, 2009-2016

\begin{tabular}{|c|c|c|c|c|c|}
\hline \multirow[t]{3}{*}{ Sex } & & \multicolumn{4}{|c|}{ Lung cancer status } \\
\hline & & \multicolumn{2}{|c|}{ Lung cancer } & \multicolumn{2}{|c|}{$\begin{array}{l}\text { Free of lung cancer } \\
\text { (controls) }\end{array}$} \\
\hline & & \multicolumn{2}{|c|}{$(n=27,017)$} & \multicolumn{2}{|c|}{$(n=129,355)$} \\
\hline & Men & 13,477 & (49.9) & 64,171 & $(49.6)$ \\
\hline & Women & 13,540 & $(50.1)$ & 65,184 & $(50.4)$ \\
\hline \multirow{6}{*}{$\begin{array}{l}\text { Age at diagnosis in cases and the cor- } \\
\text { responding age for matched controls } \\
\text { (years) }\end{array}$} & Median (q1,q3) & 70.0 & $(64.0,76.0)$ & 70.0 & $(64.0,76.0)$ \\
\hline & $<50$ & 620 & (2.3) & 3085 & (2.4) \\
\hline & $50-59$ & 2670 & (9.9) & 13,198 & $(10.2)$ \\
\hline & $60-69$ & 9381 & $(34.7)$ & 45,353 & $(35.1)$ \\
\hline & $70-79$ & 10,199 & $(37.8)$ & 48,162 & $(37.2)$ \\
\hline & $\geq 80$ & 4147 & $(15.3)$ & 19,557 & $(15.1)$ \\
\hline \multicolumn{6}{|l|}{ Educational level $^{\mathrm{a}}$} \\
\hline & Low & 10,907 & $(40.4)$ & 42,996 & (33.2) \\
\hline & Middle & 11,371 & $(42.1)$ & 52,610 & $(40.7)$ \\
\hline & High & 4350 & $(16.1)$ & 32,199 & $(24.9)$ \\
\hline & Missing & 389 & $(1.4)$ & 1550 & $(1.2)$ \\
\hline \multicolumn{6}{|l|}{ Stage at diagnosis ${ }^{\mathrm{b}}$} \\
\hline & I-II & 5922 & (21.9) & NA & NA \\
\hline & III & 5842 & $(21.6)$ & NA & NA \\
\hline & IV & 14,948 & $(55.3)$ & NA & NA \\
\hline & Missing & 305 & $(1.1)$ & NA & NA \\
\hline \multicolumn{6}{|l|}{ Histopathology } \\
\hline & SCLC & 3868 & (14.3) & NA & NA \\
\hline & NSCLC & 23,149 & $(85.7)$ & NA & NA \\
\hline \multicolumn{6}{|l|}{ Comorbid lung conditions ${ }^{\mathrm{c}}$} \\
\hline & COPD & 3383 & $(12.5)$ & 3463 & $(2.7)$ \\
\hline & Asthma & 641 & $(2.4)$ & 2861 & $(2.2)$ \\
\hline \multicolumn{6}{|c|}{ Number of filled prescriptions of antibiotics recommended for pneumonia ${ }^{\mathrm{d}}$} \\
\hline & 0 & 13,533 & $(50.1)$ & 86,787 & $(67.1)$ \\
\hline & 1 & 6732 & $(24.9)$ & 25,633 & $(19.8)$ \\
\hline & 2 & 3290 & $(12.2)$ & 9374 & $(7.2)$ \\
\hline & 3 & 1621 & (6.0) & 3761 & (2.9) \\
\hline & $\geq 4$ & 1841 & (6.8) & 3800 & (2.9) \\
\hline
\end{tabular}

Cases and controls were matched by sex, year of birth and place of residence

$q 1$ First quartile, $q 3$ Third quartile, COPD Chronic obstructive pulmonary disease, SCLC Small cell lung cancer, NSCLC Non-small cell lung cancer, NA Not applicable

Data are median (q1, q3) or $\mathrm{n}(\%)$

${ }^{a}$ Highest attained educational level the year before the index date (i.e., date of lung cancer diagnosis and the corresponding date for the matched individuals free of lung cancer), categorized by years of formal education: $\leq 9$ (low, mandatory), 10-12 (middle, upper secondary), and $\geq 13$ (high, post-upper secondary)

${ }^{\mathrm{b}}$ Based on the tumor-node-metastasis (TNM) classification system

${ }^{c}$ Within five years prior to the index date (i.e., date of lung cancer diagnosis and the corresponding date for the matched individuals free of lung cancer), based on data from the National Patient Register

${ }^{\mathrm{d}}$ Based on filled prescriptions of antibiotics recommended for the treatment of pneumonia as recorded in the Prescribed Drug Register within three years before the index date, i.e., date of lung cancer diagnosis and the corresponding date for the matched individuals free of lung cancer 
Fig. 1 The proportion of cases (individuals with lung cancer) and controls (individuals free of lung cancer) with at least one filled prescription of antibiotics recommended for the treatment of pneumonia. Presented for separate months of the observation period, overall and by history of chronic obstructive pulmonary disease. Cases and controls were matched by sex, year of birth and place of residence. Lung Cancer DataBase Sweden, 2009-2016

\section{Discussion}

\section{Main findings}

To the best of our knowledge, this is the first populationbased study using routinely collected data that has investigated patterns of pre-diagnostic use of antibiotics as a potential early indicator of lung cancer.

Compared to individuals free of lung cancer, a higher proportion of patients with lung cancer had filled at least one prescription of antibiotics within three years prior to diagnosis, and, additionally more often had a history of repeated fillings. The odds of a recent history of at least one filled prescription was approximately two times higher among patients with lung cancer compared to individuals free of lung cancer, and the magnitude of the effect size became more pronounced with increasing numbers of filled prescriptions and with proximity to the diagnosis. Also, the magnitude of the effect size was more pronounced among younger compared to older individuals, and among patients with SCC and SCLC compared to those diagnosed with adenocarcinoma. We found no evidence in support of a trend in the magnitude of the effect size by cancer stage.

\section{Interpretation and comparison with other studies}

Our findings of more frequent use of antibiotics prior to diagnosis in patients with lung cancer are for several reasons not surprising. First, pulmonary infections are not uncommon in the area of tumor growth and can present as the first symptom of a malignancy [3, 4]. Second, tumors and infected loci can initially be indistinguishable on radiographic evaluation [16]. Third, COPD and upper respiratory infections are more common in smokers at increased risk of lung cancer and individuals with COPD are often prescribed antibiotics for exacerbations [17, 18]. Taken together, our findings do not necessarily reflect an inappropriate prescribing of antibiotics. However, with as many as $7 \%$ of the patients with lung cancer in the present study having four or more treatment cycles (the maximum being 63) of antibiotics within three years prior to the diagnosis, our findings indicate improper clinical follow-up and reassessment for some patients, contrary to long-standing guidelines [19].
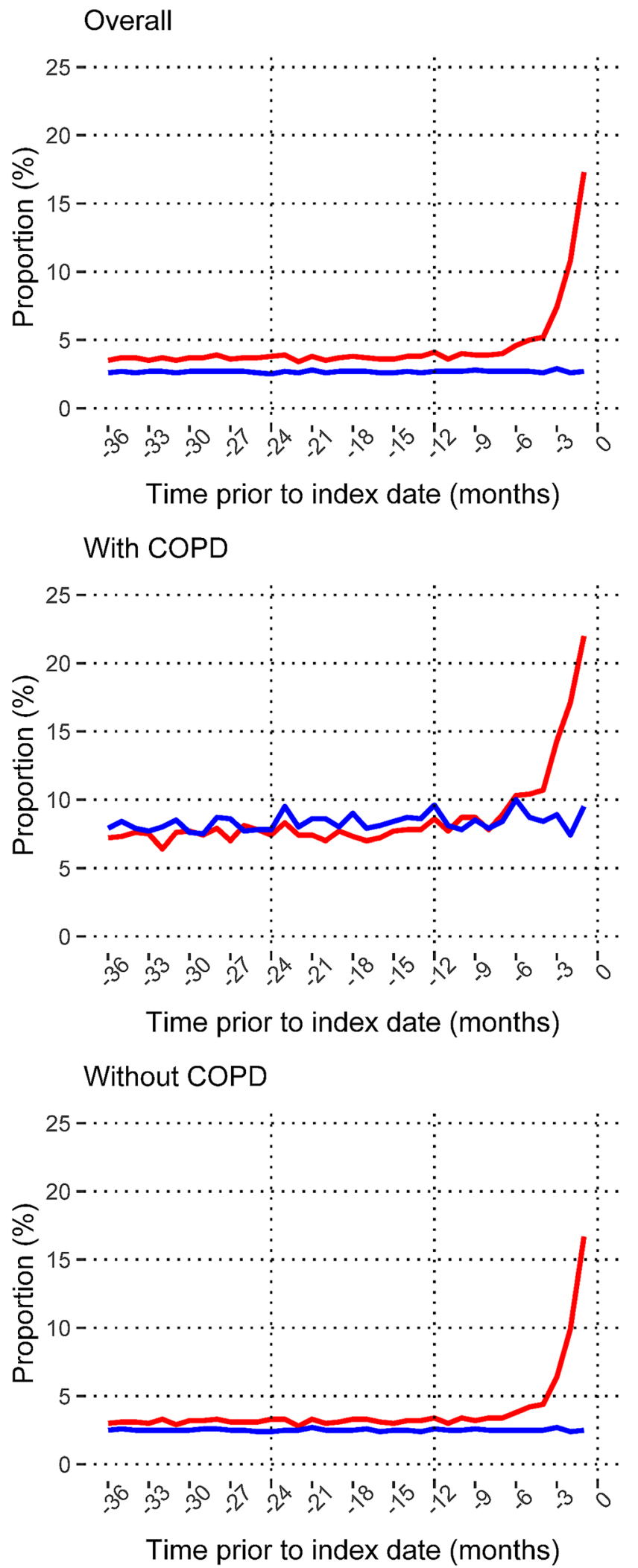

- Cases Controls 
Table 2 Odds ratios and 95\% confidence intervals for the association between a diagnosis of lung cancer and a recent history of at least one filled prescription of antibiotics recommended for the treatment of pneumonia, Lung Cancer DataBase Sweden, 2009-2016

\begin{tabular}{|c|c|c|c|c|c|c|}
\hline \multirow{3}{*}{$\begin{array}{l}\text { NSCLC, Overall } \\
\text { Overall }\end{array}$} & \multicolumn{2}{|c|}{ Exposed individuals $^{\mathrm{a}}$} & \multicolumn{4}{|c|}{ Odds ratio (95\% confidence interval) } \\
\hline & \multirow{2}{*}{$\begin{array}{l}\text { Cases } \\
11,502\end{array}$} & \multirow{2}{*}{$\begin{array}{l}\text { Controls } \\
36,472\end{array}$} & \multicolumn{2}{|c|}{ Unadjusted } & \multicolumn{2}{|c|}{ Adjusted $^{\mathrm{b}}$} \\
\hline & & & 2.04 & $(1.98-2.10)$ & 1.83 & $(1.77-1.88)$ \\
\hline \multicolumn{7}{|l|}{ Sex } \\
\hline Men & 5553 & 17,382 & 2.00 & $(1.92-2.08)$ & 1.80 & $(1.73-1.88)$ \\
\hline Women & 5949 & 19,090 & 2.08 & $(2.00-2.17)$ & 1.86 & $(1.78-1.94)$ \\
\hline \multicolumn{7}{|c|}{ Age at diagnosis (years) } \\
\hline$<50$ & 257 & 798 & 2.26 & $(1.90-2.71)$ & 2.20 & $(1.84-2.63)$ \\
\hline $50-59$ & 1136 & 3493 & 2.31 & $(2.11-2.52)$ & 2.15 & $(1.97-2.35)$ \\
\hline $60-69$ & 4120 & 13,131 & 2.08 & $(1.99-2.18)$ & 1.91 & $(1.82-2.00)$ \\
\hline $70-79$ & 4277 & 13,661 & 1.96 & $(1.88-2.05)$ & 1.72 & $(1.64-1.80)$ \\
\hline$\geq 80$ & 1712 & 5389 & 1.94 & $(1.81-2.08)$ & 1.68 & $(1.56-1.81)$ \\
\hline \multicolumn{7}{|l|}{ Educational level $^{\mathrm{c}}$} \\
\hline Low & 4447 & 11,473 & 2.06 & $(1.98-2.16)$ & 1.77 & $(1.69-1.86)$ \\
\hline Middle & 4941 & 14,800 & 2.12 & $(2.03-2.21)$ & 1.89 & $(1.81-1.97)$ \\
\hline High & 1970 & 9814 & 1.93 & $(1.81-2.06)$ & 1.81 & $(1.69-1.93)$ \\
\hline Missing & 144 & 385 & 1.90 & $(1.51-2.39)$ & 1.68 & $(1.33-2-13)$ \\
\hline \multicolumn{7}{|l|}{ SCC } \\
\hline Overall & 2966 & 8418 & 2.53 & $(2.39-2.69)$ & 2.18 & $(2.05-2.32)$ \\
\hline \multicolumn{7}{|l|}{ Sex } \\
\hline Men & 1747 & 4977 & 2.46 & $(2.31-2.63)$ & 2.13 & $(2.00-2.28)$ \\
\hline Women & 1219 & 3441 & 2.65 & $(2.48-2.84)$ & 2.26 & $(2.10-2.43)$ \\
\hline \multicolumn{7}{|c|}{ Age at diagnosis (years) } \\
\hline$<50$ & 26 & 67 & 2.97 & $(2.46-3.59)$ & 2.76 & $(2.28-3.34)$ \\
\hline $50-59$ & 250 & 599 & 2.94 & $(2.65-3.26)$ & 2.63 & $(2.37-2.93)$ \\
\hline $60-69$ & 957 & 2696 & 2.64 & $(2.46-2.84)$ & 2.33 & $(2.16-2.51)$ \\
\hline $70-79$ & 1225 & 3522 & 2.45 & $(2.28-2.62)$ & 2.07 & $(1.93-2.22)$ \\
\hline$\geq 80$ & 508 & 1534 & 2.40 & $(2.20-2.61)$ & 2.00 & $(1.84-2.19)$ \\
\hline \multicolumn{7}{|l|}{ Educational level $^{\mathrm{c}}$} \\
\hline Low & 1246 & 2906 & 2.55 & $(2.38-2.73)$ & 2.11 & $(1.97-2.27)$ \\
\hline Middle & 1264 & 3300 & 2.64 & $(2.46-2.82)$ & 2.26 & $(2.11-2.43)$ \\
\hline High & 413 & 2118 & 2.42 & $(2.22-2.63)$ & 2.17 & $(1.99-2.37)$ \\
\hline Missing & 43 & 94 & 2.34 & $(1.85-2.97)$ & 2.00 & $(1.57-2.55)$ \\
\hline \multicolumn{7}{|l|}{ Adenocarcinoma } \\
\hline Overall & 7025 & 23,299 & 1.87 & $(1.80-1.94)$ & 1.70 & $(1.64-1.77)$ \\
\hline \multicolumn{7}{|l|}{ Sex } \\
\hline Men & 3045 & 9980 & 1.80 & $(1.72-1.88)$ & 1.65 & $(1.57-1.73)$ \\
\hline Women & 3980 & 13,319 & 1.94 & $(1.85-2.02)$ & 1.75 & $(1.67-1.83)$ \\
\hline \multicolumn{7}{|c|}{ Age at diagnosis (years) } \\
\hline$<50$ & 198 & 629 & 2.16 & $(1.81-2.59)$ & 2.11 & $(1.76-2.53)$ \\
\hline $50-59$ & 747 & 2455 & 2.14 & $(1.96-2.34)$ & 2.01 & $(1.84-2.20)$ \\
\hline $60-69$ & 2667 & 8815 & 1.92 & $(1.83-2.02)$ & 1.78 & $(1.69-1.88)$ \\
\hline $70-79$ & 2482 & 8342 & 1.78 & $(1.69-1.87)$ & 1.58 & $(1.50-1.67)$ \\
\hline$\geq 80$ & 931 & 3058 & 1.74 & $(1.62-1.88)$ & 1.53 & $(1.42-1.65)$ \\
\hline \multicolumn{7}{|l|}{ Educational level $^{\mathrm{c}}$} \\
\hline Low & 2582 & 7023 & 1.89 & $(1.79-1.98)$ & 1.64 & $(1.56-1.73)$ \\
\hline Middle & 3024 & 9546 & 1.95 & $(1.86-2.05)$ & 1.76 & $(1.68-1.85)$ \\
\hline High & 1341 & 6495 & 1.79 & $(1.67-1.91)$ & 1.69 & $(1.58-1.81)$ \\
\hline Missing & 78 & 235 & 1.73 & $(1.37-2.18)$ & 1.56 & $(1.23-1.98)$ \\
\hline \multicolumn{7}{|l|}{ SCLC } \\
\hline Overall & 1982 & 6096 & 2.16 & $(2.01-2.32)$ & 1.92 & $(1.78-2.06)$ \\
\hline
\end{tabular}


Table 2 (continued)

\begin{tabular}{|c|c|c|c|c|c|c|}
\hline \multirow[t]{2}{*}{ NSCLC, Overall } & \multicolumn{2}{|c|}{ Exposed individuals $^{\mathrm{a}}$} & \multicolumn{4}{|c|}{ Odds ratio (95\% confidence interval) } \\
\hline & \multirow[t]{2}{*}{ Cases } & \multirow[t]{2}{*}{ Controls } & \multicolumn{2}{|c|}{ Unadjusted } & \multicolumn{2}{|c|}{ Adjusted $^{\mathrm{b}}$} \\
\hline \multicolumn{5}{|l|}{ Sex } & & \\
\hline Men & 887 & 2746 & 2.11 & $(1.96-2.28)$ & 1.89 & $(1.74-2.04)$ \\
\hline Women & 1095 & 3350 & 2.21 & $(2.05-2.38)$ & 1.95 & $(1.80-2.10)$ \\
\hline \multicolumn{7}{|c|}{ Age at diagnosis (years) } \\
\hline$<50$ & 46 & 120 & 2.40 & $(1.98-2.89)$ & 2.30 & $(1.90-2.79)$ \\
\hline $50-59$ & 223 & 636 & 2.43 & $(2.19-2.72)$ & 2.25 & $(2.01-2.51)$ \\
\hline $60-69$ & 727 & 2331 & 2.20 & $(2.03-2.39)$ & 1.99 & $(1.84-2.16)$ \\
\hline $70-79$ & 757 & 2322 & 2.07 & $(1.92-2.24)$ & 1.80 & $(1.66-1.95)$ \\
\hline$\geq 80$ & 229 & 687 & 2.05 & $(1.86-2.26)$ & 1.76 & $(1.59-1.94)$ \\
\hline \multicolumn{7}{|l|}{ Educational level $^{\mathrm{c}}$} \\
\hline Low & 818 & 1850 & 2.20 & $(2.03-2.38)$ & 1.86 & $(1.72-2.02)$ \\
\hline Middle & 864 & 2521 & 2.25 & $(2.09-2.44)$ & 1.98 & $(1.83-2.15)$ \\
\hline High & 277 & 1680 & 2.05 & $(1.87-2.25)$ & 1.89 & $(1.72-2.08)$ \\
\hline Missing & 23 & 45 & 2.01 & $(1.59-2.56)$ & 1.76 & $(1.38-2.26)$ \\
\hline
\end{tabular}

Cases and controls were matched by sex, year of birth and place of residence

Unexposed individuals are the reference group

NSCLC Non-small cell lung cancer, SCC Squamous cell carcinoma, SCLC Small cell lung cancer

${ }^{a}$ Individuals with at least one filled prescription of antibiotics recommended for the treatment of pneumonia as recorded in the Prescribed Drug Register within three years before the index date, i.e., date of lung cancer diagnosis and the corresponding date for the matched individuals free of lung cancer

${ }^{\mathrm{b}}$ Adjusted for sex, year of birth, place of residence, highest attained education, previous chronic obstructive pulmonary disease diagnosis, previous use of antibiotics recommended for the treatment of pneumonia, and history of any cancer

${ }^{c}$ Highest attained educational level the year before the index date (i.e., date of lung cancer diagnosis and the corresponding date for the matched individuals free of lung cancer), categorized by years of formal education: $\leq 9$ (low, mandatory), 10-12 (middle, upper secondary), and $\geq 13$ (high, post-upper secondary)

Our findings that the proportion of cases with filled antibiotic prescriptions started to increase three to four months before diagnosis as well as the pronounced effect size for being exposed in the last period prior to diagnosis indicate that early signs and symptoms of lung malignancy are commonly starting to present during this time window. Our results also corroborate the findings by Ewing et al. of an increasing frequency of primary care consultations approximately 80-100 days before confirmation of a diagnosis of lung cancer [20].

The reasons for the attenuation of the magnitude of the OR with increasing age are unclear, but may reflect a lower diagnostic intensity and underreporting of lung cancer among older individuals [21]. Also, the baseline occurrence of lung cancer is higher among older individuals, hence, a higher increase in absolute numbers of patients with lung cancer is required to yield the same relative change as for younger individuals. The more pronounced magnitudes of the effect sizes observed for SCC and SCLC compared to adenocarcinoma may reflect that these subtypes are generally more centrally located compared to adenocarcinomas and that they are associated with severe symptoms of pneumonia [22-24]. It may also reflect differences in smoking history: a higher proportion of patients with adenocarcinoma are never-smokers compared to those with SCC and SCLC $[2,25]$.

The indication of slightly attenuated magnitude of the OR for the associations between lung cancer and a history of repeated fillings for stage IV compared to stage III cancer may reflect characteristics in patients with stage III disease associated with an increased risk for infections or a presence of infection-like symptoms [26]. Stage III lung cancer is characterized by lymph node involvement or a T4-status indicating (independent of tumor size) spread to an ipsilateral lobe or invasion of heart or central parts of the lung (e.g., the carina) [27]. Patients with stage IV disease may have a small and more favorably located primary tumor without any involvement of lymph nodes, not causing infections to the same extent, and consequently, have a lower likelihood of being prescribed antibiotics before diagnosis. The descriptor specific estimates, with no trends in association for the $\mathrm{T}$ - and $\mathrm{N}$-descriptors, bring no clarity to the observed differences between stage III and stage IV. Studies investigating the causes of diagnostic delays of lung cancer have identified factors such as inconclusive chest X-ray readings, the presence of comorbid conditions, waiting times for chest 


\section{Non-small cell lung cancer, overall}

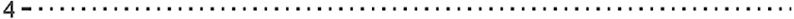

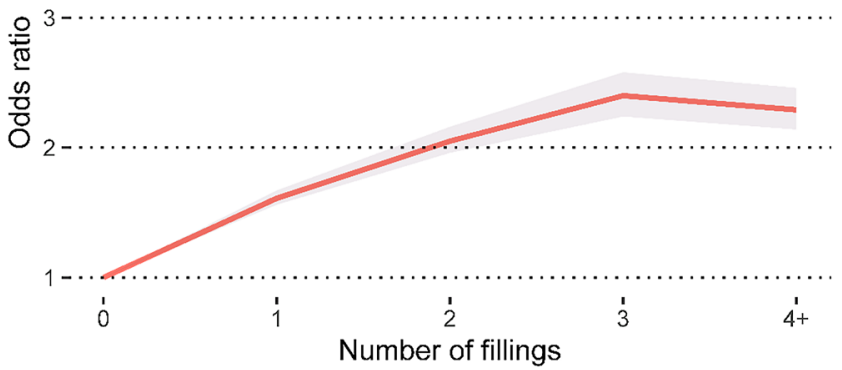

Adenocarcinoma

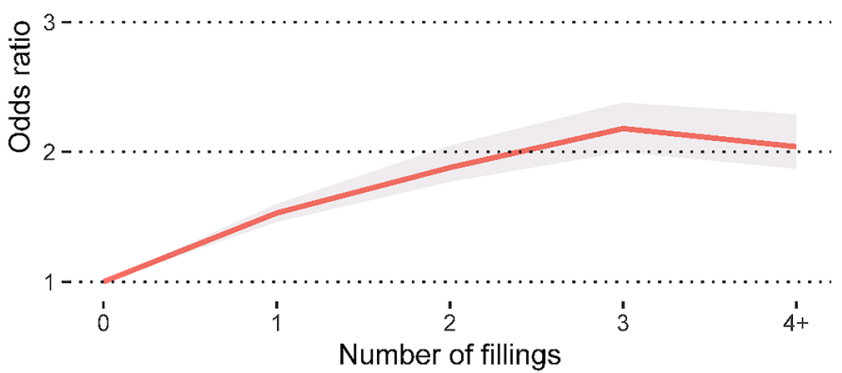

Fig. 2 Odds ratios (solid line) and 95\% confidence intervals (shaded area) for the association between a diagnosis of lung cancer and the number of recently filled prescriptions of antibiotics recommended for the treatment of pneumonia. Cases and controls were matched by sex, year of birth and place of residence. The odds ratios were

\section{Squamous cell carcinoma}

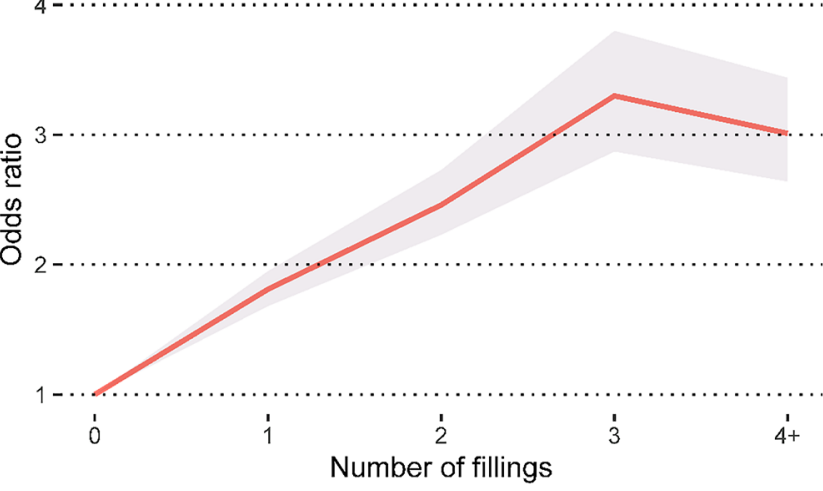

Small cell lung cancer

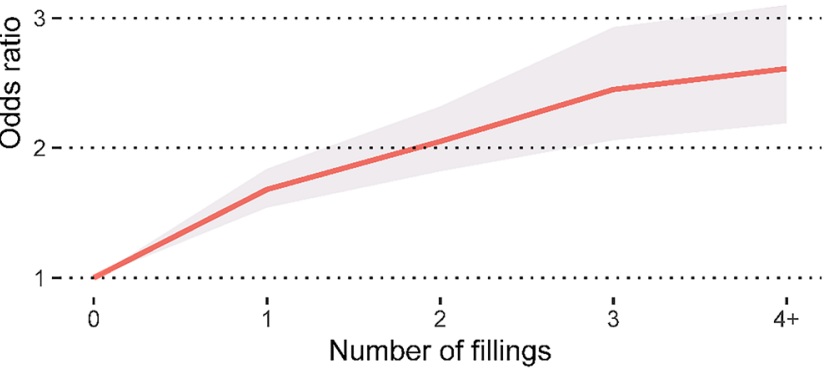

adjusted for sex, year of birth, place of residence, highest attained education, previous chronic obstructive pulmonary disease diagnosis, previous use of antibiotics recommended for the treatment of pneumonia, and history of any cancer. Lung Cancer DataBase Sweden, 2009-2016
Fig. 3 Odds ratios for the association between a diagnosis of lung cancer and a recent history of at least one filled prescription of antibiotics recommended for the treatment of pneumonia in different time intervals before diagnosis. Cases and controls were matched by sex, year of birth and place of residence. The odds ratios were adjusted for sex, year of birth, place of residence, highest attained education, previous chronic obstructive pulmonary disease diagnosis, previous use of antibiotics recommended for the treatment of pneumonia, and history of any cancer. Lung Cancer DataBase Sweden, 2009-2016

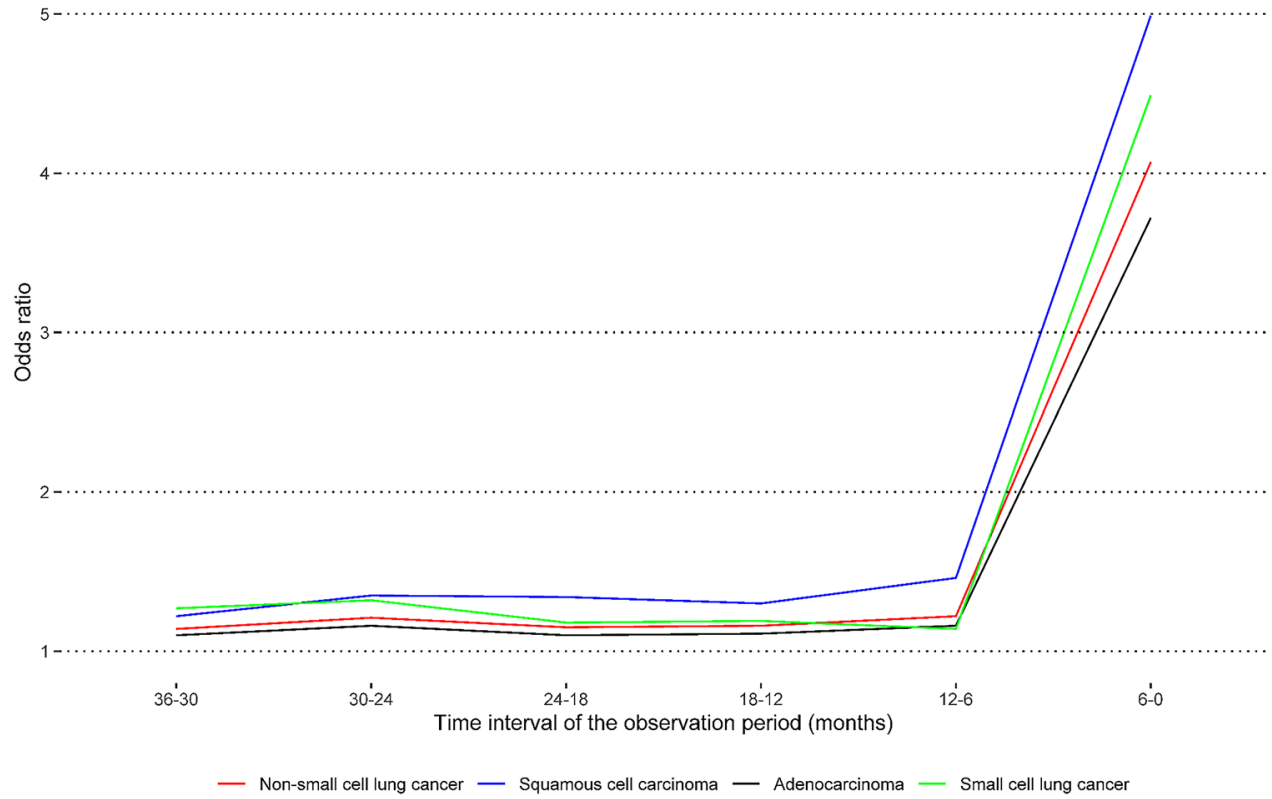


Table 3 Odds ratios and 95\% confidence intervals for the association between a diagnosis of lung cancer and a recent history of repeated fillings ( $\geq 2$ fillings) of antibiotics recommended for the treatment of pneumonia, by cancer stage at diagnosis, Lung Cancer DataBase Sweden, 2009-2016

\begin{tabular}{|c|c|c|c|c|c|}
\hline \multirow{3}{*}{ NSCLC, overall } & & \multicolumn{4}{|c|}{ Repeated fillings $(\geq 2)$} \\
\hline & & \multicolumn{2}{|c|}{ Exposed individuals $^{\mathrm{a}}$} & \multirow{2}{*}{\multicolumn{2}{|c|}{ 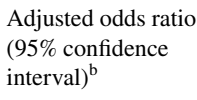 }} \\
\hline & & Cases & Controls & & \\
\hline Overall & & 5746 & 14,489 & 2.18 & $(2.10-2.27)$ \\
\hline \multicolumn{6}{|c|}{ Stage at diagnosis ${ }^{\mathrm{c}}$} \\
\hline & I-II & 1576 & 3660 & 2.28 & $(2.11-2.47)$ \\
\hline & III & 1320 & 2996 & 2.54 & $(2.33-2.77)$ \\
\hline & IV & 2796 & 7692 & 2.02 & $(1.91-2.13)$ \\
\hline & Missing & 54 & 141 & 2.10 & $(1.39-3.18)$ \\
\hline \multicolumn{6}{|l|}{ T-descriptor ${ }^{\mathrm{c}}$} \\
\hline & 1 & 1311 & 3131 & 2.29 & $(2.12-2.48)$ \\
\hline & 2 & 1594 & 4169 & 2.03 & $(1.89-2.17)$ \\
\hline & 3 & 950 & 2337 & 2.17 & $(1.99-2.38)$ \\
\hline & 4 & 1794 & 4589 & 2.17 & $(2.04-2.32)$ \\
\hline & Missing & 97 & 263 & 1.87 & $(1.45-2.40)$ \\
\hline \multicolumn{6}{|l|}{$\mathrm{N}$-descriptor ${ }^{\mathrm{c}}$} \\
\hline & 0 & 2272 & 5659 & 2.08 & $(1.96-2.21)$ \\
\hline & 1 & 419 & 1156 & 1.93 & $(1.69-2.20)$ \\
\hline & 2 & 1659 & 4190 & 2.25 & $(2.10-2.40)$ \\
\hline & 3 & 1251 & 3080 & 2.29 & $(2.13-2.47)$ \\
\hline & Missing & 145 & 404 & 1.73 & $(1.40-2.13)$ \\
\hline \multicolumn{6}{|l|}{$\mathrm{SCC}$} \\
\hline Overall & & 15,877 & 3291 & 2.75 & $(2.53-2.99)$ \\
\hline \multicolumn{6}{|c|}{ Stage at diagnosis ${ }^{\mathrm{c}}$} \\
\hline & I-II & 480 & 962 & 2.53 & $(2.17-2.95)$ \\
\hline & III & 514 & 1021 & 3.14 & $(2.71-3.65)$ \\
\hline & IV & 574 & 1264 & 2.62 & $(2.29-3.00)$ \\
\hline & Missing & 19 & 44 & 3.41 & $(1.58-7.36)$ \\
\hline \multicolumn{6}{|l|}{ T-descriptor ${ }^{\mathrm{c}}$} \\
\hline & 1 & 256 & 473 & 3.03 & $(2.72-3.38)$ \\
\hline & 2 & 501 & 1012 & 2.59 & $(2.35-2.85)$ \\
\hline & 3 & 290 & 627 & 2.75 & $(2.46-3.06)$ \\
\hline & 4 & 527 & 1151 & 2.77 & $(2.52-3.04)$ \\
\hline & Missing & 13 & 28 & 2.46 & $(1.89-3.20)$ \\
\hline \multicolumn{6}{|l|}{$\mathrm{N}$-descriptor ${ }^{\mathrm{c}}$} \\
\hline & 0 & 610 & 1313 & 2.67 & $(2.44-2.92)$ \\
\hline & 1 & 145 & 296 & 2.44 & $(2.10-2.82)$ \\
\hline & 2 & 490 & 1007 & 2.86 & $(2.60-3.15)$ \\
\hline & 3 & 306 & 585 & 2.97 & $(2.68-3.30)$ \\
\hline & Missing & 36 & 90 & 2.20 & $(1.77-2.75)$ \\
\hline \multicolumn{6}{|l|}{ Adenocarcinoma } \\
\hline Overall & & 3419 & 9324 & 2.00 & $(1.90-2.10)$ \\
\hline \multicolumn{6}{|c|}{ Stage at diagnosis ${ }^{c}$} \\
\hline & I-II & 968 & 2391 & 2.17 & $(1.97-2.40)$ \\
\hline & III & 598 & 1575 & 2.07 & $(1.83-2.33)$ \\
\hline & IV & 1826 & 5278 & 1.90 & $(1.78-2.04)$ \\
\hline & Missing & 27 & 80 & 1.80 & $(1.03-3.13)$ \\
\hline \multicolumn{6}{|l|}{ T-descriptor ${ }^{\mathrm{c}}$} \\
\hline & 1 & 920 & 2341 & 2.14 & $(1.97-2.32)$ \\
\hline & 2 & 914 & 2656 & 1.83 & $(1.70-1.97)$ \\
\hline
\end{tabular}

Table 3 (continued)

\begin{tabular}{|c|c|c|c|c|c|}
\hline \multirow{3}{*}{ NSCLC, overall } & & \multicolumn{4}{|c|}{ Repeated fillings $(\geq 2)$} \\
\hline & & \multicolumn{2}{|c|}{ Exposed individuals $^{\mathrm{a}}$} & \multirow{2}{*}{\multicolumn{2}{|c|}{$\begin{array}{l}\text { Adjusted odds ratio } \\
\text { (95\% confidence } \\
\text { interval) })^{\mathrm{b}}\end{array}$}} \\
\hline & & \multirow{2}{*}{$\begin{array}{l}\text { Cases } \\
5746\end{array}$} & \multirow{2}{*}{$\begin{array}{l}\text { Controls } \\
14,489\end{array}$} & & \\
\hline Overall & & & & 2.18 & $(2.10-2.27)$ \\
\hline & 3 & 517 & 1360 & 1.94 & $(1.76-2.13)$ \\
\hline & 4 & 1002 & 2772 & 1.96 & $(1.82-2.10)$ \\
\hline & Missing & 66 & 195 & 1.73 & $(1.35-2.24)$ \\
\hline \multicolumn{6}{|l|}{$\mathrm{N}$-descriptor ${ }^{\mathrm{c}}$} \\
\hline & 0 & 1444 & 3766 & 1.89 & $(1.77-1.98)$ \\
\hline & 1 & 216 & 710 & 1.73 & $(1.51-1.98)$ \\
\hline & 2 & 926 & 2562 & 2.03 & $(1.89-2.19)$ \\
\hline & 3 & 749 & 2054 & 2.11 & $(1.95-2.29)$ \\
\hline & Missing & 84 & 232 & 1.56 & $(1.26-1.93)$ \\
\hline \multicolumn{6}{|l|}{ SCLC } \\
\hline Overall & & 1006 & 2446 & 2.25 & $(2.04-2.49)$ \\
\hline \multicolumn{6}{|l|}{ Stage at diagnosis ${ }^{\mathrm{c}}$} \\
\hline & I-II & 47 & 116 & 1.99 & $(1.25-3.18)$ \\
\hline & III & 312 & 654 & 2.77 & $(2.29-3.35)$ \\
\hline & IV & 629 & 1642 & 2.09 & $(1.85-2.35)$ \\
\hline & Missing & 18 & 34 & 3.27 & $(1.41-7.59)$ \\
\hline \multicolumn{6}{|l|}{$\mathrm{T}_{\text {-descriptor }}{ }^{\mathrm{c}}$} \\
\hline & 1 & 119 & 264 & 2.41 & $(2.13-2.72)$ \\
\hline & 2 & 170 & 467 & 2.13 & $(1.90-2.38)$ \\
\hline & 3 & 148 & 326 & 2.28 & $(2.02-2.58)$ \\
\hline & 4 & 541 & 1322 & 2.28 & $(2.07-2.52)$ \\
\hline & Missing & 28 & 67 & 1.96 & $(1.51-2.55)$ \\
\hline \multicolumn{6}{|l|}{$\mathrm{N}$-descriptor ${ }^{\mathrm{c}}$} \\
\hline & 0 & 88 & 306 & 2.12 & $(1.89-2.38)$ \\
\hline & 1 & 49 & 111 & 1.97 & $(1.68-2.31)$ \\
\hline & 2 & 399 & 873 & 2.29 & $(2.06-2.55)$ \\
\hline & 3 & 442 & 1080 & 2.34 & $(2.11-2.60)$ \\
\hline & Missing & 28 & 76 & 1.77 & $(1.41-2.21)$ \\
\hline
\end{tabular}

Cases and controls were matched by sex, year of birth and place of residence

Unexposed individuals are the reference group

NSCLC Non-small cell lung cancer, SCC Squamous cell carcinoma, SCLC Small cell lung cancer

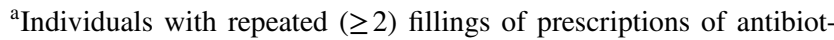
ics recommended for the treatment of pneumonia as recorded in the Prescribed Drug Register within three years before the index date, i.e., date of lung cancer diagnosis and the corresponding date for the matched individuals free of lung cancer

${ }^{\mathrm{b}}$ Adjusted for sex, year of birth, place of residence, highest attained education, previous chronic obstructive pulmonary disease diagnosis, previous use of antibiotics recommended for the treatment of pneumonia, history of any cancer, and the other TNM-descriptors in the descriptor separate analyses (e.g., if estimating effect of exposure in subgroups of T-descriptor then the estimate is adjusted for $\mathrm{N}$ - and M-descriptors)

${ }^{\mathrm{c}}$ Based on the tumor-node-metastasis (TNM) classification system 
X-ray and the absence of symptoms [16, 28]. Taken together, the findings of our study do not provide evidence that a history of repeated treatment cycles of antibiotics is related to diagnostic delays, at least not as reflected in an increased likelihood of being diagnosed with more advanced disease.

Alternative explanations for our findings include a role of antibiotics as a risk factor, possibly via an influence on the immune system or host-microbiota composition $[5,6$, 29]. However, for several reasons our findings are unlikely to be explained by a risk modifying effect of exposure to antibiotics. First, the latency period for the initiation and development of a malignancy is generally long. Second, the estimates did not change following the exclusion of individuals with filled prescriptions within the washout period of 12 months before the start of the observation period. However, we could not assess exposure before 2005, when the PDR was launched.

\section{Strengths and limitations}

The strengths of our study included the use of data from nationwide Swedish population-based registers of high completeness and quality that minimized the risk of selection bias and misclassification bias of exposures and outcomes. The completeness and validity of prescription data in the PDR is very high, mainly because the transfer of information from community pharmacies is almost exclusively automated [13].

Several limitations need mentioning. No information was available for the indication of the prescribed antibiotics. While we restricted the analyses to antibiotics recommended for the treatment of pneumonia, it cannot be ruled out that some prescriptions were for other infections. However, because respiratory infections are one of the most common indications for antibiotic use [30], this is likely to have resulted in no or only a minor misclassification of exposure. Also, assuming a higher risk of respiratory infections in patients with lung cancer (for which the majority are current or former smokers) compared to the general population, any misclassification is likely to be more common in controls and would have biased the results toward the null. The absence of data of actual use by the patient was not a concern because the aim of this study was not to examine exposure to antibiotics as a risk factor per se. Any non-differential misclassification of the exposure or the outcome would have biased the ORs toward the null. Although most pneumonia diagnosis are made in a primary care setting, such information based on ICD coding is not available in the NPR or in the other data sources used for the purpose of the present study. However, all prescriptions made in primary care are included in the PDR, once a prescription is filled.
Because information on smoking history was available for lung cancer patients only, smoking could not be included in the adjusted models. However, information on COPD diagnosis retrieved from the NPR was included in the logistic regression models.

Our findings are likely to be generalisable to settings with similar health care systems and guidelines for the management of pneumonia and lung cancer.

\section{Conclusions}

We found that a diagnosis of lung cancer was associated with an increased likelihood of recent pre-diagnostic fillings of antibiotic prescriptions. The likelihood became more pronounced with a greater number of fillings and with proximity to the diagnosis, further supporting the notion that infection represents an early sign of lung cancer. We found no evidence that repeated treatment cycles were associated with a diagnostic delay as reflected by cancer stage at diagnosis. Our findings further underscore the importance to rule out lung cancer following pneumonia treatment, especially in patients with a history of repeated treatment cycles.

Supplementary Information The online version contains supplementary material available at https://doi.org/10.1007/s10552-021-01413-5.

Acknowledgments The study was funded by grants from the Swedish Cancer Society (15-0804 and 18-0689). The project was made possible by the continuous reporting by Swedish clinicians to the NLCR and the work by the Swedish National Lung Cancer register steering group. We would also like to thank Sarah Burkill for the language check and Pär Karlsson for his valuable comments on the statistical analyses.

Authors' contribution LL performed the statistical analysis and prepared the first draft of the manuscript. All authors have contributed to the study design, interpreting the results, and contributed to writing the manuscript. All authors approved the final version of the manuscript. The corresponding author attests that all listed authors meet authorship criteria and that no others meeting the criteria have been omitted.

Funding Open access funding provided by Karolinska Institute. The study was funded by grants from the Swedish Cancer Society (15-0804 and 18-0689).

Data availability Data may be obtained from the registries named in the methods section and are not publicly available. The authors are, according to the law, not authorized to make the data publicly available.

Code Availability On request.

\section{Declarations}

Conflict of interest Lukas Löfling, Shahram Bahmanyar, and Helle Kieler are employees at the Centre for Pharmacoepidemiology at Karolinska Institutet, which receives funding from several entities (pharmaceutical companies, regulatory agencies, and contract research organizations). Mats Lambe declares stock ownership in Pfizer and As- 
tra Zeneca. Gunnar Wagenius has no conflict of interest to declare. The funding bodies had no role in the data collection and analysis and were not involved in the interpretation of results, writing, revision, or approval of the manuscript.

Ethical approval This study was approved by the Regional Ethical Review Boards in Uppsala and Stockholm (record number: 2012/1162 31/4, 2016/1137-32, 2017/445-32, 2017/2026-32).

Consent to participate This study only includes register data, and the research was done without patient involvement.

Consent for publication This study only includes register data, and the research was done without patient involvement.

Open Access This article is licensed under a Creative Commons Attribution 4.0 International License, which permits use, sharing, adaptation, distribution and reproduction in any medium or format, as long as you give appropriate credit to the original author(s) and the source, provide a link to the Creative Commons licence, and indicate if changes were made. The images or other third party material in this article are included in the article's Creative Commons licence, unless indicated otherwise in a credit line to the material. If material is not included in the article's Creative Commons licence and your intended use is not permitted by statutory regulation or exceeds the permitted use, you will need to obtain permission directly from the copyright holder. To view a copy of this licence, visit http://creativecommons.org/licenses/by/4.0/.

\section{References}

1. Ebell MH, Thai TN, Royalty KJ (2018) Cancer screening recommendations: an international comparison of high income countries. Public Health Rev 39:7

2. Löfling $\mathrm{L}$ et al (2019) Clinical characteristics and survival in nonsmall cell lung cancer patients by smoking history: a populationbased cohort study. Acta Oncol 58(11):1618-1627

3. Karl-Gustav Kölbeck and H. Almer. Regionalt Vårdprogram Lungcancer [Regional Care Program - Lung Cancer]. 2018 [cited 2019 20190114]; Available from: http://www.viss.nu/Handlaggni ng/Vardprogram/Andning/Lungcancer/.

4. National Health Service (NHS). Lung cancer symptoms. 2019 20190815 [cited 2019 190827]; Available from: https://www.nhs. uk/conditions/lung-cancer/symptoms/\#.

5. Zhang H, Garcia Rodriguez LA, Hernandez-Diaz S (2008) Antibiotic use and the risk of lung cancer. Cancer Epidemiol Biomarkers Prev 17(6): 1308-1315

6. Friedman GD et al (2009) Screening pharmaceuticals for possible carcinogenic effects: initial positive results for drugs not previously screened. Cancer Causes Control 20(10):1821-1835

7. Regional Cancer Centre - Uppsala Örebro, Lungcancerregistret: Lungcancerrapport för diagnosår 2013-2017. 2018: Uppsala, Sweden.

8. Socialstyrelsen - Swedish National Board of Health and Welfare. The Swedish Cancer Register. 201920190509 191122]; Available from: https://www.socialstyrelsen.se/en/statistics-and-data/regis ters/register-information/swedish-cancer-register/.

9. Skatteverket - The Swedish Tax Agency. The Swedish Population Register. 2020 [cited 2020 200604]; Available from: https://www. skatteverket.se/privat/folkbokforing/attvarafolkbokford/folkbokfor ingsdatabasen.4.3810a01c150939e893f16fe2.html.

10. Willén L et al (2019) Educational level and management and outcomes in non-small cell lung cancer. A nationwide populationbased study. Lung Cancer 131:40-46
11. Regional Cancer Centre - Uppsala Örebro. Swedish National Lung Cancer Register. 2017 170913]; Available from: http://www. cancercentrum.se/vast/cancerdiagnoser/lunga-och-lungsack/kvali tetsregister/.

12. Socialstyrelsen -Swedish National Board of Health and Welfare. The Swedish National Patient Register 201920190520 191122]; Available from: https://www.socialstyrelsen.se/en/statistics-anddata/registers/register-information/the-national-patient-register/.

13. Socialstyrelsen - Swedish National Board of Health and Welfare. The Swedish Prescribed Drug Register. 201920191018 191122]; Available from: https://www.socialstyrelsen.se/statistik-och-data/ register/alla-register/lakemedelsregistret/.

14. Statistiska Centralbyrån (SCB) -Statistics Sweden. Longitudinal Integration Database for Health Insurance and Labour Market Studies (LISA). 2016 20171026]; Available from: https://www. scb.se/contentassets/f0bc88c852364b6ea5c1654a0cc90234/ dokumentation-av-lisa.pdf.

15. American Joint Committee on Cancer, AJCC cancer staging manual: 7th edition. 7th ed. 2010, New York, USA: Springer Verlag New York Inc.

16. Bjerager $\mathrm{M}$ et al (2006) Delay in diagnosis of lung cancer in general practice. The British J Gen Prac 56(532):863-868

17. Durham AL, Adcock IM (2015) The relationship between COPD and lung cancer. Lung Cancer 90(2):121-127

18. Labaki WW, Han MK (2017) Antibiotics for COPD exacerbations. The Lancet Respiratory medicine 5(6):461-462

19. Stralin K et al (2008) Management of adult patients with community-acquired pneumonia. Evidence-based guidelines from the Swedish infectious diseases association. Lakartidningen 105(38):2582-2587

20. Ewing $\mathrm{M}$ et al (2016) Increased consultation frequency in primary care, a risk marker for cancer: a case-control study. Scand J Prim Health Care 34(2):205-212

21. Barlow $\mathrm{L}$ et al (2009) The completeness of the Swedish cancer register: a sample survey for year 1998. Acta Oncol 48(1):27-33

22. American Cancer Society. What is lung cancer? 2016 200204]; Available from: http://www.cancer.org/content/cancer/en/cancer/ lung-cancer/about/what-is.html.

23. Hoffman PC, Mauer AM, Vokes EE (2000) Lung cancer. Lancet 355(9202):479-485

24. Mendoza TR et al (2019) Assessment of baseline symptom burden in treatment-naive patients with lung cancer: an observational study. Support Care Cancer 27(9):3439-3447

25. Stone $\mathrm{S}$ et al (2000) Antibiotic prescribing for patients with colds, upper respiratory tract infections, and bronchitis: a national study of hospital-based emergency departments. Ann Emerg Med 36(4):320-327

26. Mayo Clinic. Swollen lymph nodes. 201920191009 20200127]; Available from: https://www.mayoclinic.org/diseases-conditions/ swollen-lymph-nodes/symptoms-causes/syc-20353902.

27. Goldstraw $P$ et al (2016) The IASLC lung cancer staging project: proposals for revision of the TNM stage groupings in the forthcoming (Eighth) edition of the TNM classification for lung cancer. J Thorac Oncol 11(1):39-51

28. Cassim S et al (2019) Patient and carer perceived barriers to early presentation and diagnosis of lung cancer: a systematic review. BMC Cancer 19(1):25

29. Maddi A et al (2019) The microbiome and lung cancer. Journal of Thoracic Disease 11(1):280-291

30. Aabenhus R et al (2017) Clinical indications for antibiotic use in Danish general practice: results from a nationwide electronic prescription database. Scand J Prim Health Care 35(2):162-169

Publisher's Note Springer Nature remains neutral with regard to jurisdictional claims in published maps and institutional affiliations. 